\title{
Editorial 51
}

\author{
Eric Scerri ${ }^{1}$
}

Published online: 23 September 2015

(C) Springer Science+Business Media Dordrecht 2015

This latest offering is a combination of a special issue on nano-chemistry which will be introduced by the guest editor Jean-Pierre Llored. The remainder of the issue contains various papers that are briefly described by myself below.

Jan Boeyens has been a prolific author in the foundations of chemistry for many years, with a large number of articles and books to his credit. In the article appearing in the present issue, he presents a grand vision involving hypercomplex algebra which he claims can bring together aspects of quantum mechanics and relativity theory. Through this approach Boeyens provides a new understanding of electron spin and offers a challenge to the all-pervasive approach involving the linear combination of atomic orbitals in chemistry and physics.

The second article is in the field of chemical education and addresses the important question of how much emphasis should be placed on conceptual understanding as opposed to the application of memorized facts and algorithms. Drawing on research in cognitive psychology the authors, Hartman and Nelson, discuss the importance of fostering 'automaticity' in connection with long-term memory and the relevance of these issues for chemical education.

The last of the articles in the varia section deals with group theory and symmetry, subjects that have a large bearing in so many areas of modern chemistry. Michael Downward presents a simplification to the usual group theoretical approaches by introducing a system that uses just a minimal set of generators for three dimensional groups. Among other beneficial features the new approach permits a replacement of the large number of tables that are usually encountered in the Schoenflies approach.

Eric Scerri

scerri@chem.ucla.edu

1 Department of Chemistry and Biochemistry, University of California, Los Angeles (UCLA),

Los Angeles, CA, USA 